A. Weisbecker, M. Burmester \& A. Schmidt (Hrsg.): Mensch und Computer 2015 Workshopband, Stuttgart: Oldenbourg Wissenschaftsverlag, 2015, S. 583-588.

\title{
Ein Use Case Management Repository zur Unterstützung der Normungsarbeit
}

\author{
Marion Gottschalk, Mathias Uslar
}

FuE Bereich Energie, Architekturentwicklung und Interoperabilität, OFFIS e.V.

\section{Zusammenfassung}

Die Anzahl an Möglichkeiten, das alltägliche Leben eines Menschen jeglichen Alters zu unterstützen, steigt durch die stetige Weiterentwicklung von Informations- und Kommunikationstechnologien und der Möglichkeit diese Technologien miteinander zu verknüpfen. Als kurzes Beispiel sei hier das Smartphone genannt, welches sich in den letzten Jahren zu einer in vielen Bereichen einsetzbaren Technologie entwickelt hat. So können mit Smartphones verschiedenste Komponenten und Systeme im menschlichen Umfeld angesprochen und gesteuert werden, z.B. die Heizungssteuerung. Damit die Verknüpfung von neuen Systemen und Technologien zur Unterstützung des alltäglichen Lebens reibungslos funktioniert, werden Normen und Standards benötigt. Allerdings ist es schwierig, vorab alle Normen zu definieren, da die Unterstützungsmöglichkeiten zahlreich sind. Daher soll der Normungsprozess durch die Beschreibung und Sammlung von möglichen Anwendungsfällen (engl. Use Cases) unterstützt werden, um Schnittstellen zu erkennen und mittels Integrationsprofilen nach IHE zu definieren. Dazu wird in diesem Beitrag das Use Case Management Repository vorgestellt.

\section{Motivation}

Das alltägliche Leben eines Menschen jeglichen Alters kann durch die Bereitstellung von Informationen aus seiner mittelbaren und unmittelbaren Umgebung unterstützt werden. Für die Verarbeitung dieser Informationen kommen immer mehr Informations- und Kommunikationstechnologien (IKT) zum Einsatz, deren technische Entwicklung rasant fortschreitet. Dies sind z.B. Technologien wie Smartphones und smarte Fernsehgeräte, welche eine immer größere Rolle im alltäglichen Leben spielen. Aufgrund dieser Entwicklungen erschließen sich weitere Einsatzbereiche, Menschen unterwegs oder in ihrem häuslichen Umfeld zu unterstützen, engl. Ambient Assisted Living (DKEa, 2014). Eine Unterstützungsmöglichkeit stellt z.B. die Integration eines Sicherheitssystems im eigenen Haushalt dar. Dieses Assistenzsystem kann Menschen in ihrem häuslichen Umfeld unterstützen, in dem z.B. alle Türen und Fenster beim Verlassen des Hauses durch Aktuatoren automatisch verschlossen werden. Neben dem Schutz vor unbekannten Dritten kann das Sicherheitssystem aber auch weitere Funktionen im Haushalt übernehmen, z.B. mittels Bewegungsmelder die Licht- oder Hei- 
zungsanlage steuern. Um diese Funktionen umzusetzen und anwendergerecht einzustellen, bedarf es den Einsatz von Smartphones und weiteren IKT (Eberhardt \& Eichelberg, 2011). Mit diesem Beispiel soll ein kleiner Einblick über die Vielzahl an Komponenten und Systeme gegeben werden, die für die Umsetzung nur eines Assistenzsystems relevant sind. $\mathrm{Zu}$ Bedenken ist, dass diese Komponenten bzw. Systeme auch für weitere Systeme im Umfeld von Ambient Assisted Living (AAL) relevant sind, wie z.B. Healthmonitoring, Notfallerkennungssysteme, etc. Daher ist es wichtig, dass diese Komponenten und Systeme nahtlos kommunizieren und zusammenarbeiten, um Informationen auszutauschen, d.h. diese Komponenten und Systeme sind interoperabel zu gestalten. Da nicht alle Komponenten und Systeme von einem einzigen Hersteller bereitgestellt werden, werden kompatible Schnittstellen für den Informationsaustausch benötigt. Ein Beispiel hierfür ist der Kommunikationsstandard WLAN (Wireless Local Area Network), welcher z.B. den Informationsaustausch zwischen Smartphone und Sicherheitssystem ermöglicht. Daher ist die Entwicklung gemeinsamer Normen und Standards relevant, um Komponenten und Systeme miteinander zu verknüpfen. Um den Vorgang der Normung zu unterstützen, muss allerdings vorab bekannt sein, was genau genormt werden soll. Hierfür ist es sinnvoll, sich ein vollständiges Bild von den Funktionen einer durch AAL unterstützten Umgebung zu verschaffen. Da es sich hier um eine sehr komplexe Domäne mit vielen Akteuren (Komponenten und Systemen) verschiedener Hersteller und unterschiedlichen Anwendern handelt, ist es sinnvoll durch Anwendungsfälle (engl. Use Cases) mögliche Szenarien für die Umgebungen, die durch AAL Systeme unterstützt werden, zu beschreiben. Daher werden in diesem Beitrag die Use Case Methodik, der Einsatz von Integrationsprofilen und das Use Case Management Repository (UCMR) vorgestellt, um die Arbeit in der Normung zu unterstützen.

\section{Grundlagen: Anwendungsfälle \& Integrationsprofile}

Anwendungsfälle dienen der funktionalen Beschreibung eines bzw. mehrerer Systeme. Ein Nutzen durch Anwendungsfälle kann allerdings erst verzeichnet werden, wenn diese ebenfalls einheitlich beschrieben und damit für verschiedene Experten verständlich sind. Dafür wird die Beschreibung eines Anwendungsfalls in mehrere Schritte unterteilt und beinhaltet somit verschiedene Perspektiven einer funktionalen Beschreibung, welche für unterschiedliche Experten relevant sind. Diese Darstellung basiert auf dem Template IEC/PAS 62559 (IEC/PAS 62559, 2008) für Anwendungsfälle, welches bereits in der Smart Grid Domäne erfolgreich eingesetzt wurde, um Funktionen dieser Domäne darzustellen (Santodomingo, 2014). Dabei wurden Anwendungsfälle mit verschiedenen Experten entwickelt und gesammelt, um eine erste Übersicht zur Smart Grid Domäne zu erstellen. Daraus konnte zudem ein Architekturmodell entwickelt werden, mit dem Anwendungsfälle visualisiert werden (DKE, 2010). Das Template IEC/PAS 62559 soll in weiteren Domänen, wie z.B. AAL (DKE, 2014) und Smart Cities (DKEb, 2014 \& Gottschalk, 2015), eingesetzt werden, um eine einheitliche Entwicklung voranzubringen. Das Template beinhaltet an erster Stelle eine allgemeine Beschreibung des Anwendungsfalls, welche für die späteren Anwender der AAL Komponenten also auch für das Management der Hersteller der AAL Komponenten verständlich ist. Dieser allgemeine Part kann durch die Verknüpfung mit Referenzen und Grafiken unterstützt und 
erweitert werden, sodass weitere Informationen für die Leser bereitstehen. Für die Domänenexperten besteht dann die Möglichkeit, die vorhandenen Anwendungsfälle durch die detaillierte Beschreibung von Szenarien und einzelnen Schritte zu erweitern, um genauere Schnittstellenanforderungen zu identifizieren. Dabei werden die Akteure, welche die AAL Komponenten, externe Komponenten aber auch die Anwender darstellen, verknüpft. Ferner können IKT-Experten festhalten, welche Daten zwischen diesen Akteuren ausgetauscht werden müssen, wodurch die Beschreibung der Schnittstellen der einzelnen Akteure konkretisiert wird.

Eine weitere Möglichkeit Schnittstellen der Akteure detailliert zu beschreiben, bieten Integrationsprofile, welche alle Schnittstellen und die davon betroffenen Akteure eines Anwendungsfalls darstellen. Der Einsatz von Integrationsprofilen hat sich bereits in der „Integrating the Healthcare Enterprise (IHE)“ Initiative bewährt (Eichelberg, Rölker-Denker und Hein, 2014) und daher soll das Template IEC/PAS 62559 um diese Inhalte erweitert werden. Integrationsprofile beinhalten eine allgemeine Beschreibung mit Grafik, eine Auflistung von möglichen Transaktionen der involvierten Akteure, eine Beschreibung des Datenflusses mit Grafik und einer Beschreibung von ethischen und rechtlichen Rahmenbedingungen. Ein Teil

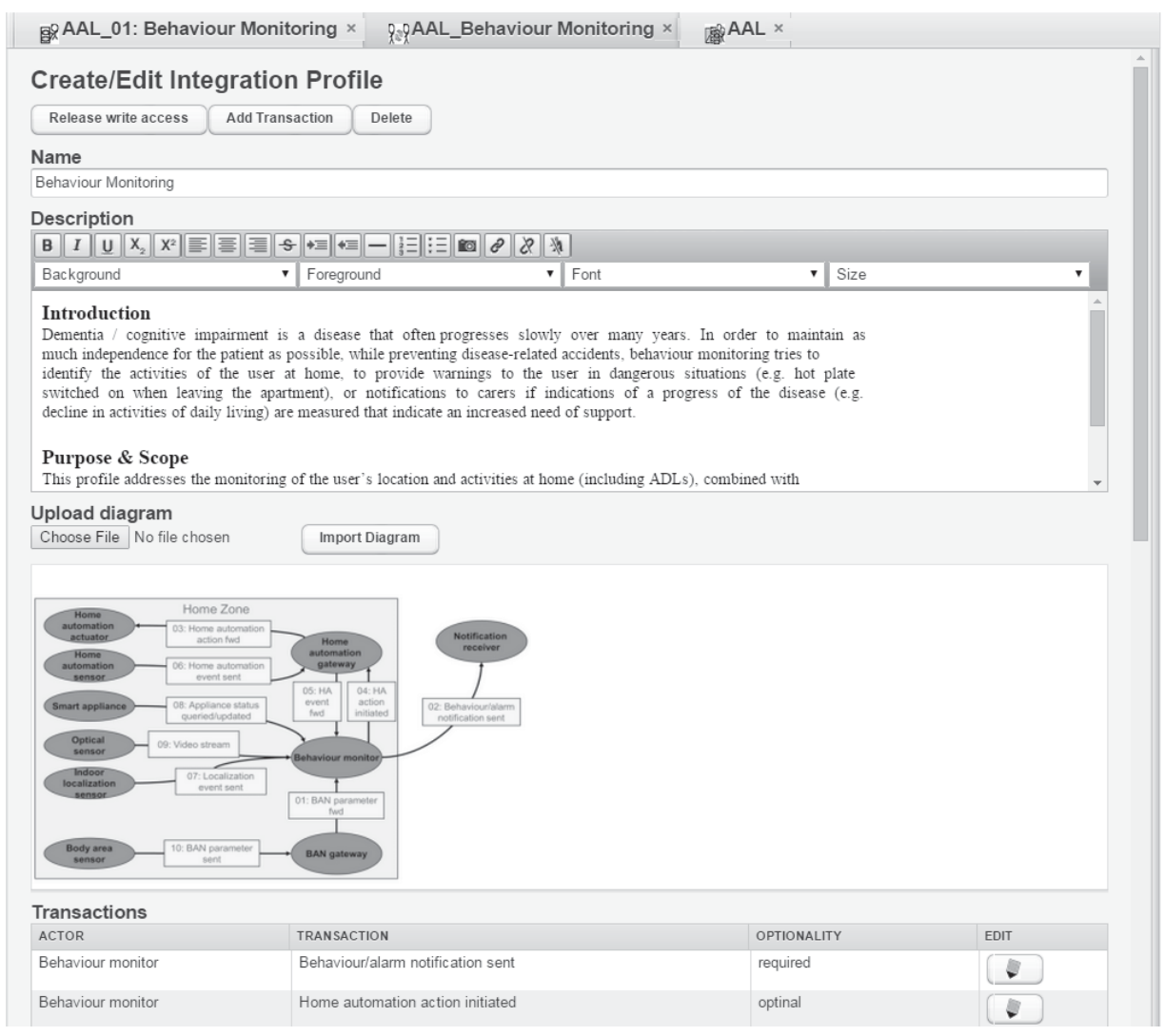

Abbildung 1: Integrationsprofile 
des Integrationsprofile-Templates ist in Abbildung $1 \mathrm{zu}$ sehen. Die verwendeten Daten im Integrationsprofil sind dem „AAL Joint Programme“ (Eichelberg, Rölker-Denker und Helm, 2014) entnommen und nicht vollständig abgebildet. Die im Integrationsprofil aufgelisteten Schnittstellen werden durch einzelne Transaktionen im Detail beschrieben, um Schnittstellenanforderungen genau zu spezifizieren. Daher beinhalten die Transaktionen eine detaillierte Beschreibung der beteiligten Akteure, indem eine Role, die Wichtigkeit und die Anforderungen der einzelnen Akteure dargestellt werden. Außerdem werden Protokolle für die Kommunikation bzw. den Datenaustauch beschrieben und Sicherheitsaspekte, die diese Schnittstelle einhalten muss, aufgezeigt.

\section{Use Case Management Repository- UCMR}

Mithilfe des oben genannten IEC/PAS 62559 Templates wird die einheitliche Beschreibung der Anwendungsfälle ermöglicht. Der zweite wichtige Aspekt ist nun, diese Anwendungsfälle alle Beteiligten zur Verfügung zu stellen und eine kollaborative Erarbeitung der Anwendungsfälle zu ermöglichen. Dies kann über ein gemeinsames Repository, einem Use Case Management Repository (UCMR), gewährleistet werden. Ein UCMR bietet die Möglichkeit, Anwendungsfälle zu erstellen, die von Experten mit verschiedenen Sichtweisen auf das System erarbeitet und verifiziert werden. Ferner lassen sich die Anwendungsfälle gruppieren und kombinieren, sodass Zusammenhänge zwischen den verschiedenen Anwendungsfällen dargestellt werden können. Das oben aufgezeigte Integrationsprofil in Abbildung 1 ist bereits in das entwickelte UCMR eingebunden. Anhand der Liste von Anwendungsfällen und den darin beschriebenen Schnittstellen zwischen Akteuren können Normen ausgewählt und der Normungsprozess verbessert werden. Zusätzlich können bereits vorhandene Normen und Standards in den Anwendungsfällen annotiert werden, sodass diese Information im UCMR allen Beteiligten zur Verfügung steht. Denn die Normen stellen zukünftig eine Grundlage zur Herstellung von AAL Komponenten dar, um Komponenten bzw. Systeme zu produzieren, die interoperabel zu anderen Systemen von Drittherstellern sind. Dies erleichtert den Anwendern von AAL Komponenten die Kombination verschiedener Systeme, um ihre eigene AAL Umgebung zu erschaffen, die auf ihre speziellen Bedürfnisse angepasst ist. Auch die Kommunikation nach außen zu Ärzten oder anderen Dienstleistungsanbieter lässt sich durch die Verwendung gemeinsamer Normen realisieren. Aber nicht nur die Kommunikation zu Ärzten und Dienstleistungsanbietern für AAL Komponenten lässt sich durch Normen vereinfachen, auch andere angrenzende Domänen, wie z.B. das Smart Grid oder eMobility, können in die Kommunikation mit eingebunden werden. Denn Akteure, die in der Domäne AAL eine Rolle spielen, können ebenfalls weitere Aufgaben in anderen Domänen übernehmen. Da wären z.B. Aktuatoren für das Öffnen und Schließen von Fenstern. Diese Aktuatoren werden in der Domäne AAL eingesetzt, um Sicherheitsaspekte zu erfüllen (siehe Abschnitt 1. Motivation) und um Anwendern mit körperlichen Beeinträchtigungen das Öffnen und Schließen eines Fensters zu erleichtern. In der Domäne Smart Grid werden dieselben Aktuatoren vom Smart Meter angesteuert, um die Belüftung eines Hauses so energieeffizient wie möglich zu gestalten. Die Überschneidungen verschiedener Domänen kann ebenfalls durch das UCMR berücksichtigt und dargestellt werden, sodass Akteure, welche in verschiedenen Domänen 
interagieren, nicht mehrfach verwaltet werden müssen. Die domänenübergreifende Betrachtung von Akteuren erleichtert außerdem die Normungsarbeit für übergreifende Schnittstellen. Mit diesen Funktionalitäten bietet das UCMR die Möglichkeit, eine strukturierte Sichtweise auf komplexe und domänenübergreifende Systeme $\mathrm{zu}$ erhalten und die Normungsarbeit in diesen Bereichen zu unterstützen. Das UCMR ist als Webanwendung für alle an der Normung beteiligten Experten erreichbar und ermöglicht mit einer Import- und Exportfunktion das Austauschen von Informationen zwischen verschiedenen Anwendungen. Das WebInterface des UCMRs ist in Abbildung 2 zu sehen. In der Abbildung werden Informationen zu den Domänen Smart Grid, AAL und Smart City dargestellt und sind somit für alle Experten aus verschiedenen Domänen verfügbar und erlauben einen domänenübergreifenden Informationsaustausch (Gottschalk, 2014). Am linken Rand von Abbildung 2 lässt sich erkennen, wie die einzelnen Bereiche, AAL, Smart Grid und Smart City, untergliedert sind. Der erste Ordner „Use Cases“ beinhaltet alle Anwendungsfälle, die in einem Bereich beschrieben sind. Die anderen drei Ordner stellen Daten bereit, die in der Beschreibung von Anwendungsfällen benötigt werden. Diese Daten sind die Akteure, Funktionen und Anforderungen, welche gemeinsam von den Anwendern des UCMRs erstellt und daraufhin in der Beschreibung der Anwendungsfälle verwendet werden. Dies erlaubt vorab eine einheitliche Definition der Komponenten eines Anwendungsfalls und ermöglicht das Wiederverwenden dieser Daten, wodurch die Beschreibung der Anwendungsfälle konsistent gehalten werden kann. Auf der rechten Seite in Abbildung 2 werden die ausgewählten Daten und Anwendungsfälle in Tabs dargestellt.

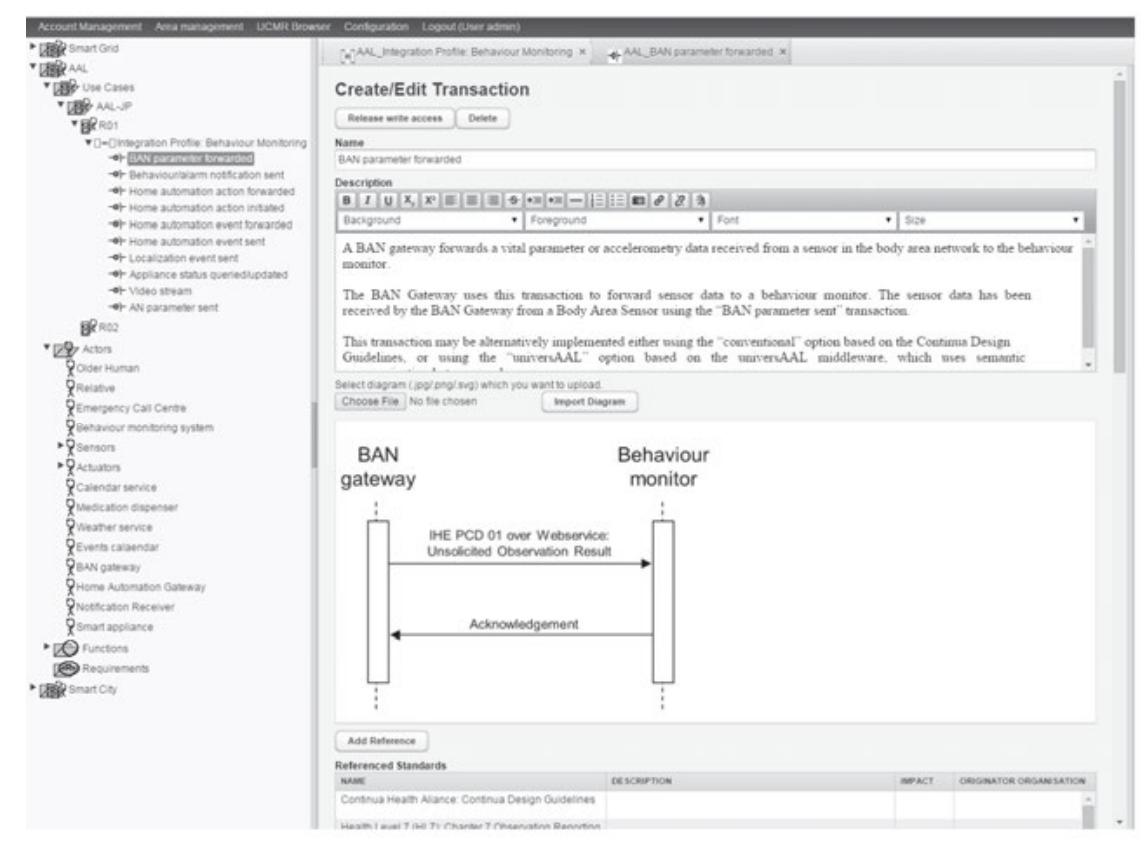

Abbildung 2: Use Case Management Repository 


\section{$4 \quad$ Fazit}

Durch den Einsatz des UCMR werden Informationen zu komplexen Systemen und Wissen zu bereits vorhandenen Lösungen zwischen Experten ausgetauscht, um die einheitliche Beschreibung von komplexen, domänenübergreifenden Systemen und die Entwicklung von Normen zu unterstützen. Gerade in der Domäne AAL steigt die Nachfrage nach interoperablen Systemen, um das Wohnen im Eigenheim bis ins hohe Alter zu ermöglichen. Wie das Anwendungsbeispiel in der Motivation gezeigt hat, werden bereits für ein Assistenzsystem verschiedenste Komponenten und Systeme zur Realisierung benötigt, welche wiederum in weiteren Assistenzsystemen zum Einsatz kommen könnten. Daher wird eine vollständige Betrachtung der Domäne AAL und deren angrenzenden Domänen gefordert, um eine interoperable Infrastruktur zu entwickeln.

\section{Danksagung}

Die Forschung, Anpassung und Entwicklung des UCMRs durch das OFFIS konnte aufgrund der Förderung des Bundesministeriums für Wirtschaft und Energie im Projekt „UC4AAL“ durchgeführt werden.

\section{Literaturverzeichnis}

Borghoff, U. M. \& Schlichter, J. (1998). Rechnergestützte Gruppenarbeit - Eine Einführung in Verteilte Anwendungen. 2. Auflage, Springer.

DKE (2010). Die deutsche Normungs-Roadmap E-Energy / Smart Grid.

DKE (2014). Die deutsche Normungs-Roadmap AAL (Ambient Assisted Living) Version 2.

DKE (2014). Die deutsche Normungs-Roadmap Smart City Version 1.0.

Eberhardt, B., \& M. Eichelberg. (2011). AAL-Anwendungsszenarien, In: VDE Verlag.

Eichelberg, M., Rölker-Denker, L. und Hein, A. (2014). Recommendations on Interoperability, Specifications and Standards. In 7. Dtsch. AAL-Kongress mit Ausstellung.

Eichelberg, M., Rölker-Denker, L. und Helmer, A. (2014). Action Aimed at Promoting Standards and Interoperability in the Field of AAL Deliverable D2 AAL Use Cases and Integration Profiles.

Englert, H. und Uslar, M. (2012): Europäisches Architekturmodell für Smart Grids-Methodik und Anwendung der Ergebnisse der Arbeitsgruppe „Referenzarchitektur “des EU Normungsmandats M/490, VDE-Kongress 2012

Gottschalk, M. Göring, A. und Uslar, M (2014). Applying the Use Case Methodology to Smart Cities. In VDE-Kongress 2014.

Gottschalk, M. und Uslar, M. (2015). Supporting the Development of Smart Cities using a Use Case Methodology. In 24th International World Wide Web Konferenz.

IEC/PAS 62559 (2008): IntelliGrid Methodology for Developing Requirements for Energy Systems.

Santodomingo, R., Uslar, M., Göring, A., Gottschalk, M., Nordström, L., Saleem, A. und Chenine, M. (2014). SGAM-based methodology to analyze Smart Grid solutions. In IEEE EnergyCon 2014.

\section{Kontaktinformationen}

M.Sc. Marion Gottschalk (gottschalk@offis.de), OFFIS, Escherweg 2, 26121 Oldenburg. 